\title{
Evaluation of graphic effects embedded image compression
}

\author{
Chanintorn Jittawiriyanukoon ${ }^{1}$, Vilasinee Srisarkun² \\ ${ }^{1}$ Graduate School of Advanced Technology Management, Assumption University, Thailand \\ ${ }^{2}$ Martin de Tours School of Management and Economics, Assumption University, Thailand
}

\begin{tabular}{l}
\hline \hline Article Info \\
\hline Article history: \\
Received Feb 15, 2020 \\
Revised Jun 6, 2020 \\
Accepted Jun 18, 2020 \\
\hline Keywords: \\
Computer vision \\
Frequency domain \\
Graphic effects \\
Grayscale image \\
Image compression \\
Spatial domain
\end{tabular}

\begin{abstract}
A fundamental factor of digital image compression is the conversion processes. The intention of this process is to understand the shape of an image and to modify the digital image to a grayscale configuration where the encoding of the compression technique is operational. This article focuses on an investigation of compression algorithms for images with artistic effects. A key component in image compression is how to effectively preserve the original quality of images. Image compression is to condense by lessening the redundant data of images in order that they are transformed cost-effectively. The common techniques include discrete cosine transform (DCT), fast Fourier transform (FFT), and shifted FFT (SFFT). Experimental results point out compression ratio between original RGB images and grayscale images, as well as comparison. The superior algorithm improving a shape comprehension for images with grahic effect is SFFT technique.
\end{abstract}

Copyright $\left(C_{0} 2020\right.$ Institute of Advanced Engineering and Science. All rights reserved.

\section{Corresponding Author:}

Chanintorn Jittawiriyanukoon, Assumption University,

Thailand.

Email: pct2526@yahoo.com

\section{INTRODUCTION}

Digital image [1] is the collection of pixels, outlines, RGB colors, and domains to represent any physical substances. It is a $3 \mathrm{D}$ or $2 \mathrm{D}$ representation. Number in each pixel denotes the intensity of illumination on an x-y plane. The reflection of light is detected by human eyes, machines, etc. A graylevel or grayscale digital image is an example of artlessly compressed picture where whole colors are grayness (dullness) [2]. In other words, gray means the RGB combination with whole identical intensity. The benefit for designating digital images from their authentic RGB colors is that slighter data to be collected for each pixel. The intensity can only be deposited as eight bits ranging from zero to 255 possible representations. Grayscale images [3] are essential, for many capture and display tasks, they are completely acceptable, and it is unnecessary to opt more complex color images. Especially, as we only expect appearance and structure like shape and effects grayness is substantial [4]. One transformation of image processing is a compression in which a foreseen size of authentic data is condensed. It is beneficial, particularly for a raster image that possesses a massive data to pack for manageable transfer and spatial domain [5].

For a presentation of image compression, the RGB color data will not reflect some characteristics such as lines or edges. There is exclusive for an edge in shade that is unlikely detectable in grayslevel images. For various applications, we do not need RGB, as it is considered as redundancy or unwanted data or noise [6]. To find significant edges based on both chrominance and luminance [7] is tedious as it is inflexible to confirm if the redundant RGB data are not cooperative for points of focus. In graylevel images, compression algorithms are simple to visualize as preference can be the $2 \mathrm{D}$ spatial domain plus one dimension for illumination then they can be represented by a 3D visualization. In RGB, color spectrum visualization is complex as there are further dimensions that human eyes can perceive [8]. We can consider color variables to identify image intensity; however it eventually brings us back to a graylevel image instead. With parallel processing and high-speed computers [9], it is potential to convert color-to-grayscale 
pixel-by-pixel of a raster image in nanoseconds. The processing time required to compress some helpful data from a facial image is quicker. It is beneficial to save processing time by focusing on useful parts of images and ignoring unwanted color variables. Besides, there are several methods to transform color-to-grayscale as presented in [10]. In computer vision, a color-to-grayscale conversion based on genetic algorithm is presented in [11] to retain the characteristics of the color images, such as lightness and luminosity. A grayscale conversion algorithm based on modulation domain is applied to color images and is proposed by [12]. The grayscale output is developed by a blend of three colors where the combination coefficients are based on a quadratic parameter.

\section{IMAGE COMPRESSION ALGORITHMS}

With the increasing demand in applications of digital image, there is a consistent research in the area of image compression algorithms [13]. The intention is to characterize an original RGB image in the lowest number of bits without dropping the critical content. Image holds three basic types of content within: value, non-relation, and redundancy. Valuable part is the information we need to defend. Non-relational part is the extra information, which is beyond the scope of implication. Redundant part can be regenerated from other corresponding contents [14]. Human vision judges image after decompression. The transfer of compressed images is affected by real-time system [15] while the space domain problem is tolerant. There are two main forms of compression approaches, lossy and lossless types. The former one is a common practice, in which the information of an initial image will partly get missing (distorted) but this approach maximizes compression ratio on the overhead of decompression precision. These applications incorporate the re-production of a raster image, and the transfer of compressed digital images on communication networks. While the latter one, the squeezed image must contain a precise model of the authentic image. Their applications include biological data, e-documents, camera visualization, satellite images [16-18], and medical archives, in which the distorted information can lead to fault analytics.

\subsection{Discrete cosine transform (DCT)}

It is fortunate that the human vision can reimburse for the malformation in compressed images, not like numeric or text data. DCT [19] is one of lossy compression algorithms, which consumes as low bandwidth as expected. At the compression step, the quantization requires bit length for individual image while lessening redundancy. It makes this approach attain high compression ratio with a trade-off between the quality of image and the bandwidth required. Regarding applications, a critical ratio is manageable provided that the distortion is somehow acceptable. The 8 by 8 blocks DCT operation is generally associated to discrete Fourier transform (DFT), orthogonally separates the original image, and transforms it from spatial to frequency domain. Besides, the technique is an origin of common compression like jpg format. The encoding 2D ( $I \times J$ image) DCT compression is shown in the following (1).

$$
F(u, v)=\sqrt{\frac{2}{J}} \sqrt{\frac{2}{I}} C(u) C(v) \sum_{x=0}^{I-1} \sum_{y=0}^{J-1} f(x, y) \cos \left[\frac{\pi u}{2 I}(2 x+1)\right] \cos \left[\frac{\pi v}{2 J}(2 y+1)\right]
$$

For $u=0, \ldots, I-1, v=0, \ldots, J-1, C(d)=1 / \sqrt{2}$ for $d=0$, and $C(d)=1$ for $d \neq 0$ (where $I=J=8$ ). The (2) shows the decoding $2 \mathrm{D}$ ( $I \times J$ image) DCT compression.

$$
f(x, y)=\sqrt{\frac{2}{J}} \sqrt{\frac{2}{I}} \sum_{u=0}^{I-1} \sum_{v=0}^{J-1} C(u) C(v) F(u, v) \cos \left[\frac{\pi u}{2 I}(2 x+1)\right] \cos \left[\frac{\pi v}{2 J}(2 y+1)\right]
$$

For $x=0, \ldots, I-1, y=0, \ldots, J-1, C(d)=1 / \sqrt{2}$ for $d=0$, and $C(d)=1$ for $d \neq 0$ (where $I=J=8)$.

\subsection{Fast fourier transform (FFT)}

The FFT [20] is derived from discrete Fourier transform technique. It is to analyze then transform a spatial domain into frequency domain in image compression. Once images are in frequency domain, they are much quicker in processing than to execute in the spatial domain. Because it is simple to compute frequency (convolution) embedded in an image by solving differential equations. In other words, the FFT can simplify the complex intensity computation into straightforward multiplication. The calculation to encode the $2 \mathrm{D}$ ( $I \times J$ image) transformation in the frequency domain is listed in (3).

$$
F(u, v)=\sum_{x=0}^{I-1} \sum_{y=0}^{J-1} f(x, y) e^{-2 \pi j\left(\frac{u x}{I}+\frac{v y}{J}\right)}
$$


The (4) demonstrates the decoding 2D ( $I \times J$ image) FFT compression.

$$
f(x, y)=\frac{1}{I J} \sum_{x=0}^{I-1} \sum_{y=0}^{J-1} F(u, v) e^{2 \pi j\left(\frac{u x}{I}+\frac{v y}{J}\right)}
$$

\subsection{Shifted FFT (SFFT)}

In general, the zero frequency of the spectrum locates at the front and back component, while the highest frequency locates at the midpoint. That allows us to shift the frequencies. However, it is not supporting for a screen as the low frequencies are locating in two ends. Then to reallocate the low (or zero) frequency to the center can make a screen more attractive and this is what we anticipate. To attain 2D (I x J image) SFFT transformation [21], this is the shifting encoder which can be defined as presented in (5).

$$
F\left(u-u_{0}, v-v_{0}\right)=F^{-1}\left[f\left(x-x_{0}, y-y_{0}\right)\right]=F(u, v) e^{2 \pi j\left(\frac{u x_{0}}{I}+\frac{v y_{0}}{J}\right)}
$$

The (6) denotes the decoding 2D ( $I \times J$ image) shifted FFT transform.

$$
f\left(x-x_{0}, y-y_{0}\right)=F^{-1}\left[F\left(u-u_{0}, v-v_{0}\right)\right]=f(x, y) e^{-2 \pi j\left(\frac{u x_{0}}{I}+\frac{v y_{0}}{J}\right)}
$$

Let $\mathrm{x}_{0}$ and $\mathrm{y}_{0}$ be the frequency shift we want to display. Given that SFFT in $I$ by $J$ size of $2 \mathrm{D}$ image, our considered image has $I$ rows and $J$ columns. For example, we define a frequency value for the $\mathrm{x}$ and $\mathrm{y}$ axes between -50 and 50, when our image is of size $100 \times 100$ and it centers the image to be in the midpoint.

\subsection{Graphic effects}

To enhance feature of grayscale images we can add a visual support in the appearance of a graphic novel, such as shadows, backgrounds, contrast, sharpness, or brightness. Graphic effects are what human calls art today. They are not all identical but their harmony concludes the practice of visualization. Besides, in eLearning we can create a graphic storyboard from these novels. The objective is to make grayscale images as if they were drawn with artistic style. Integration of arts in education [22, 23] helps achieve the learning process. Researchers also believe that arts can be a reform tool in education to improve exam scores and to motivate learners. This paper focuses on five artistic effects which are marker, grow-edge, cut-out, photocopy, and plastic-wrap as displayed in Figure 1.

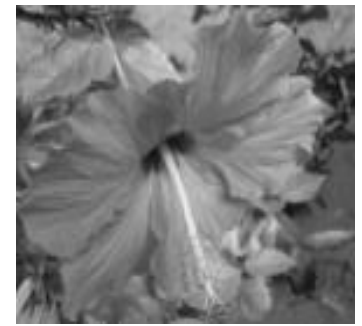

(a)

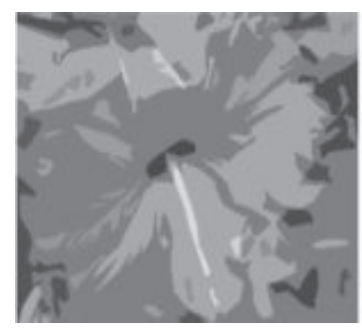

(d)

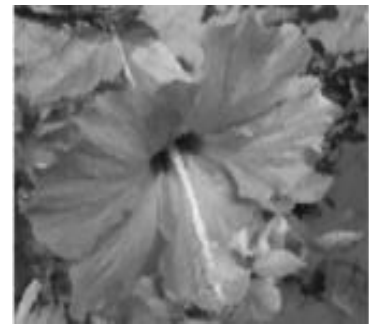

(b)

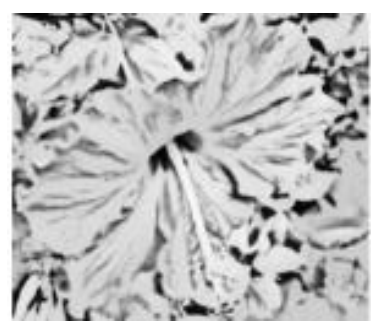

(e)

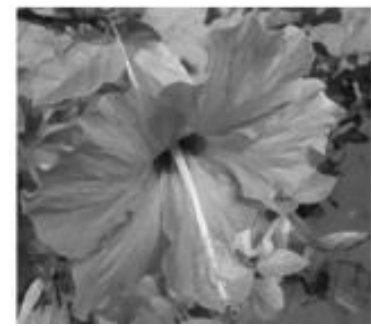

(c)

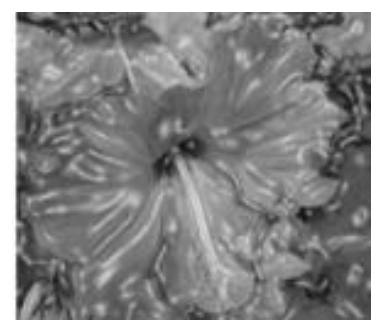

(f)

Figure 1. Graphic effects, (a) non-effect, (b) marker, (c) glow-edge, (d) cut-out, (e) photocopy, (f) plastic-wrap 


\section{EVALUATION AND ANALYSIS}

Comparative study of DCT, FFT and SFFT algorithms is performed based on illustrative visualization. We consider three RGB images and three associated grayscale images for comparing these compression algorithms. The compression rate on experimental images depends on BT. 709 graylevel converter [24]. It is commonly utilized as a standard conversion for HDTV. The luminance computation is based on Gray_Color $=0.21 * \mathrm{R}+0.72 * \mathrm{G}+0.07 * \mathrm{~B}$. Results (colormap) are visualized in Matlab which avails engineering built-in functions and setting for image analytics. The considered images are ahead transformed into grayscale as shades change accordingly between RGB entries and then artistic effects [25] are applied.

\subsection{Compression rate (CR)}

The $\mathrm{CR}$ is to calculate the performance in image compression by measuring the reducible size of compressed image (the higher the better). In lossy compression, it is to remove unnecessary data in images to minimize the image size (or resolution) then CR reflects image quality. Note that although the original image size is suffered, it is simply invisible to human vision. From each of the following three images, we summarize the size pre and post compression as well as CR figure as depicted in Table 1.

Table 1. Three sample images

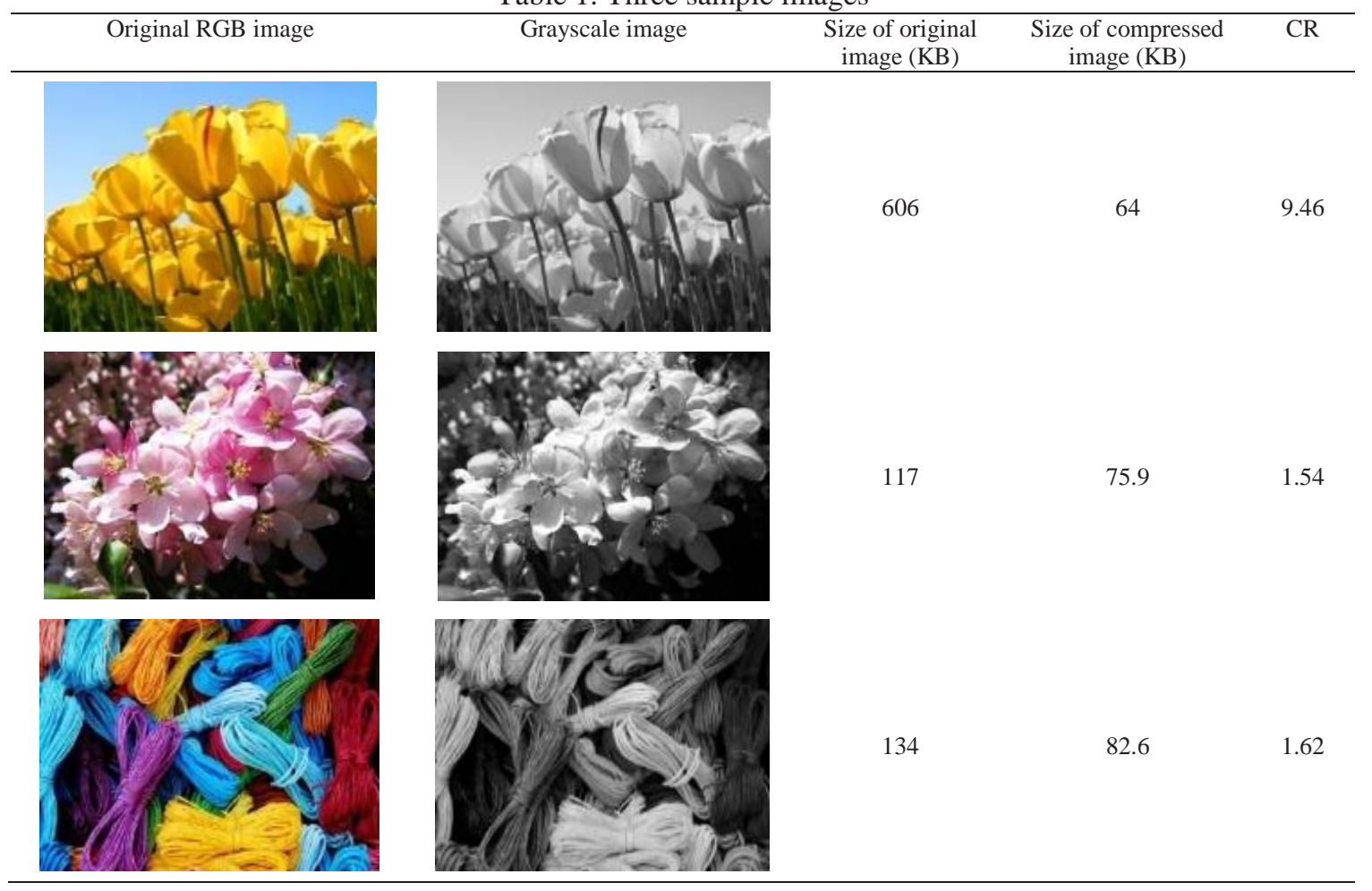

\subsection{Colormap visualization}

The method employs colors in the engineering image visualization [26]. Specially, it presents visual map that we can perceive by mixing up colors of a scalar unit. Colormaps are arranged by where they are best practice. Colors are represented in integers ranging between 0 and 255 analysis.

\subsection{D frequency domain}

A colormap appearance does not reflect alterations in value in brightness (luminance). Nonetheless, in a 3D frequency plot, shade per se which reflects changes in luminance, is vibrant to communicate transforms in shape [27]. In practice, we can avoid getting the luminance alterations in the colormap affect the shading changes by controlling the colormap to lively colors.

A grayscale colormap is useful for human vision regarding shape knowledge. It also reflects the isoluminant colormap which is supportive to comprehend insights. The luminance map is perceived so accurately and effectively by humans. Results of graysclae colormaps for original image and other five images with artistic effect are displayed in Figure 2 to Figure 7(a), respectively. It is apparent that regarding a shape comprehension the SFFT algorithm outperforms other algorithms. 
The isometric 3D plots contribute the display of a frequency spectrum. It is developed in a wire-mesh graphic. The original image and other artistic effect embedded images are visualized as presented in Figure 2 to Figure 7(b), correspondingly. The plot provides significant comprehension in the frequency spectrom and statistical function per se. It also helps convey a good visual of the 3D intensity form. It is supportive that the frequency spectrum of the SFFT algorithm aligns well and reflects the structure of considered images.

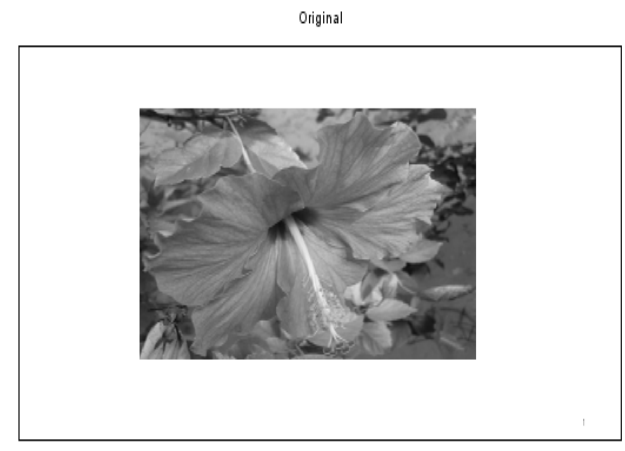

FFT

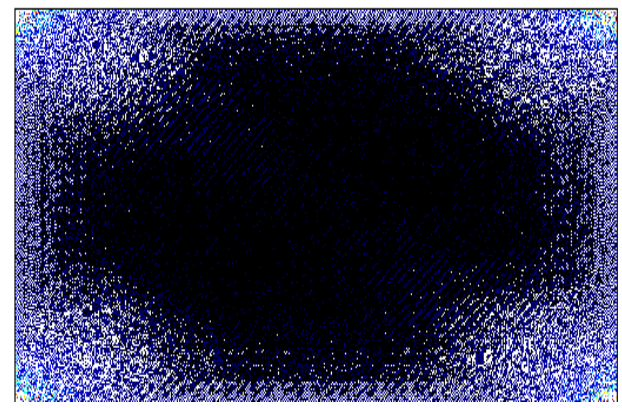

DCT

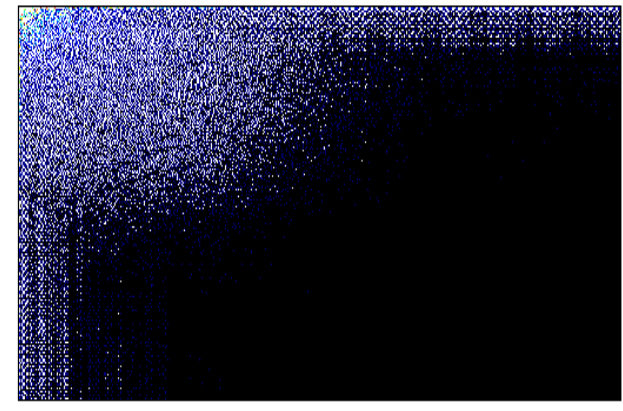

SFFT

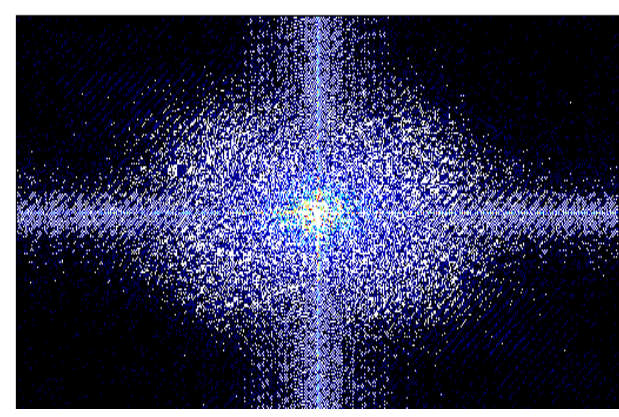

(a)
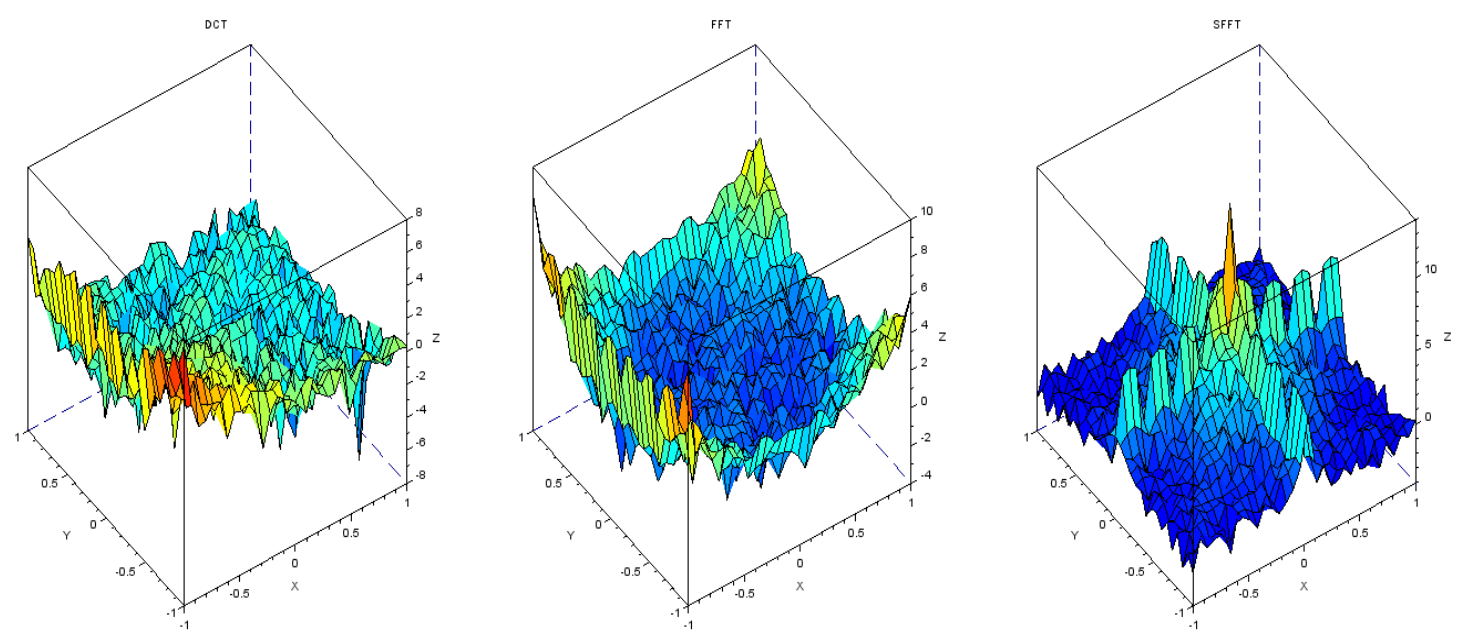

(b)

Figure 2. Original image results, a) Original image without effect (top-left) and three colormaps of DCT algorithm (top-right), FFT algorithm (bottom-left), SFFT algorithm (bottom-right), b) Frequency domain visualization of DCT algorithm (left), FFT algorithm (center), and SFFT algorithm (left) 


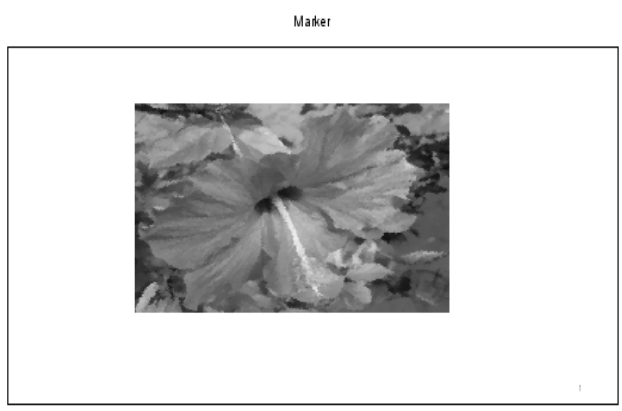

FFT

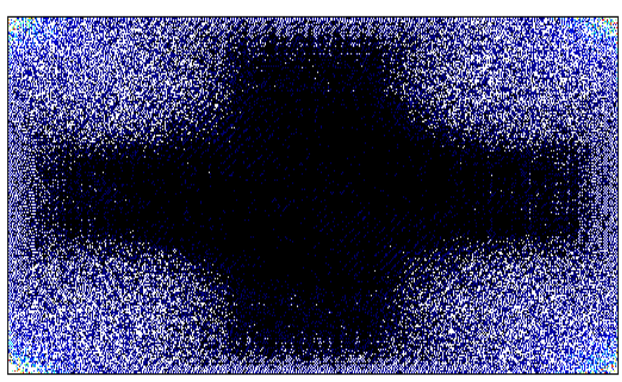

(a)
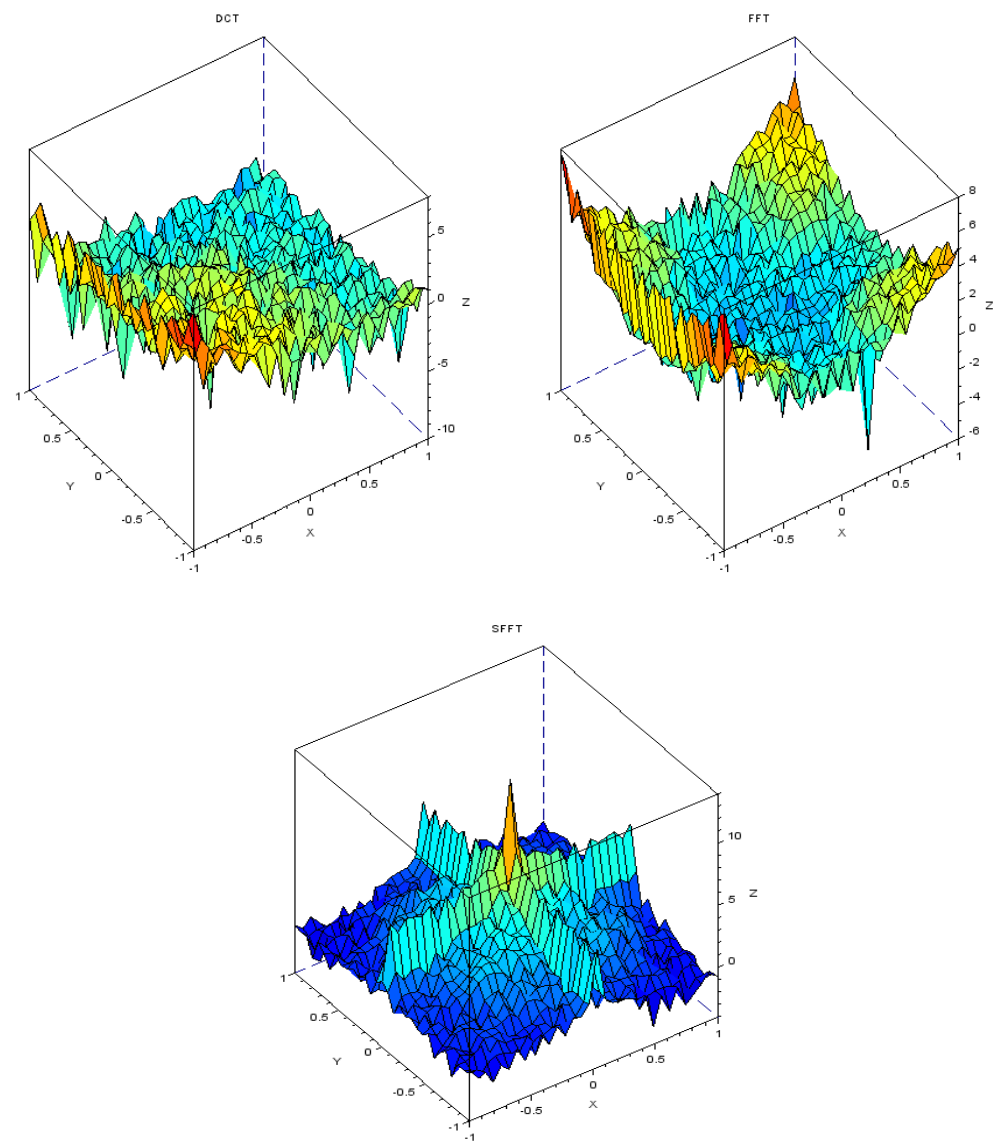

(b)

Figure 3. Results of image with marker effect, (a) Image with marker effect (top-left) and three colormaps of DCT algorithm (top-right), FFT algorithm (bottom-left), SFFT algorithm (bottom-right), (b) Frequency domain visualization of DCT algorithm (left), FFT algorithm (right), and SFFT algorithm (bottom) 


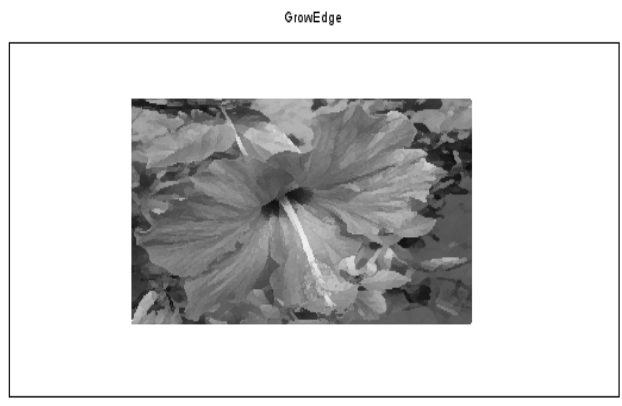

FFT

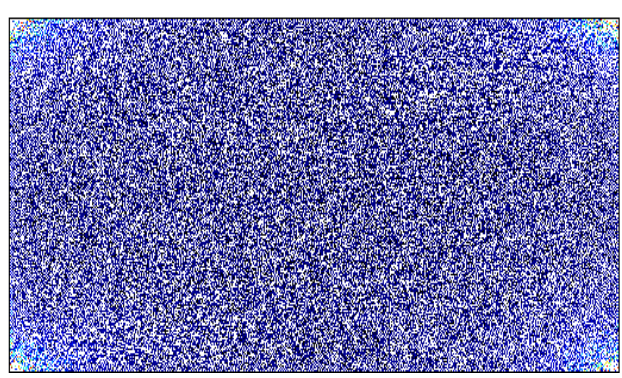

(a)
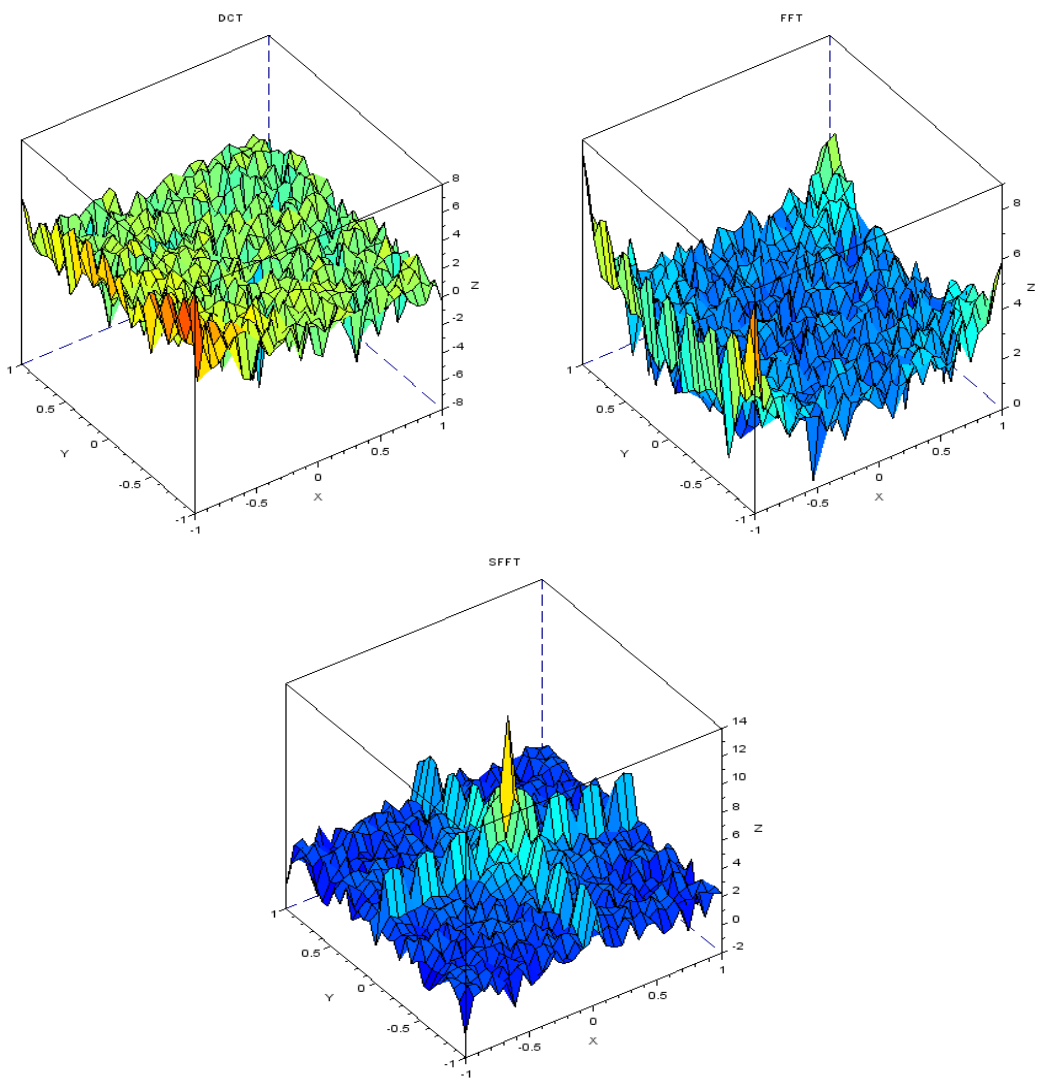

(b)

Figure 4. Results of image with grow-edge effect, (a) Image with grow-edge effect (top-left) and three colormaps of DCT algorithm (top-right), FFT algorithm (bottom-left), SFFT algorithm (bottom-right), (b) Frequency domain visualization of DCT algorithm (left), FFT algorithm (right), and SFFT algorithm (bottom) 


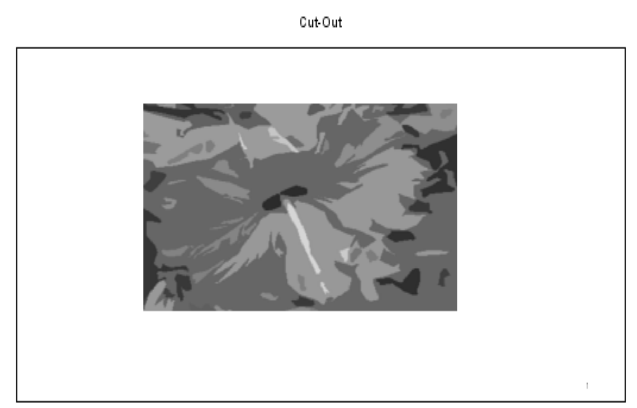

FFT

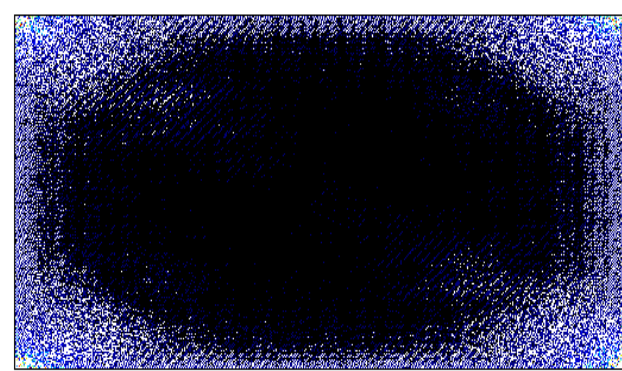

(a)
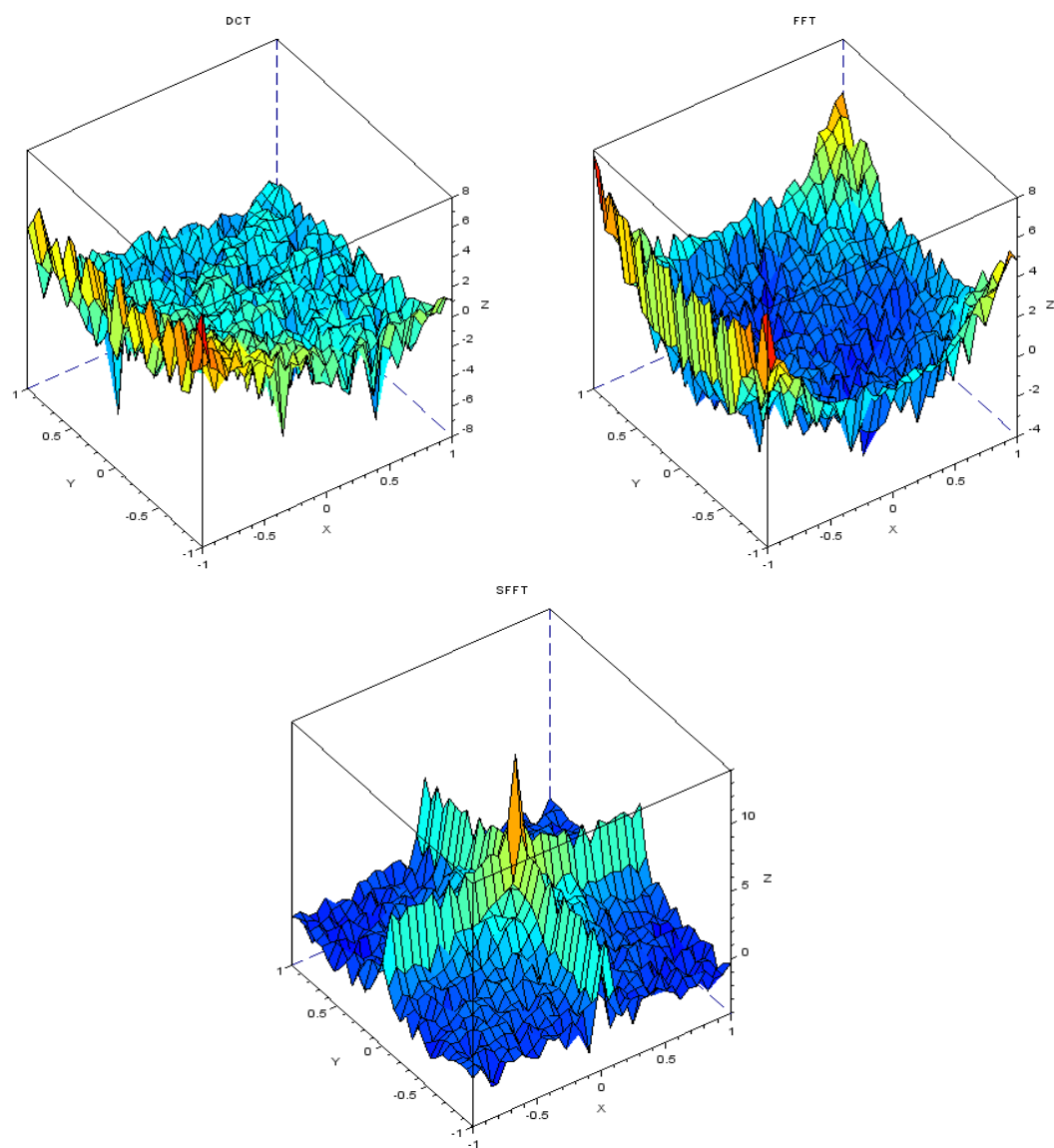

(b)

Figure 5. Results of image with cut-out effect, a) Image with cut-out effect (top-left) and three colormaps of DCT algorithm (top-right), FFT algorithm (bottom-left), SFFT algorithm (bottom-right), b) Frequency domain visualization of DCT algorithm (left), FFT algorithm (right), and SFFT algorithm (bottom) 


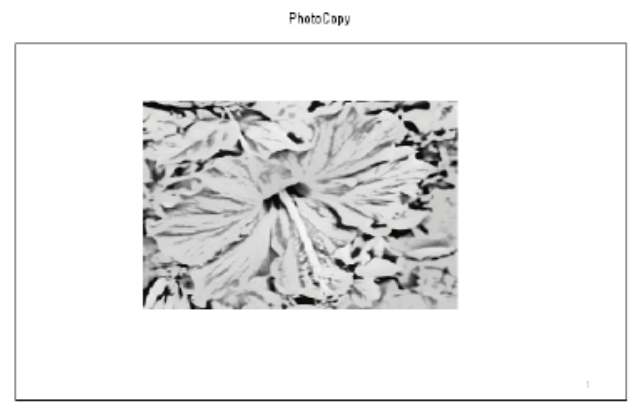

FFT

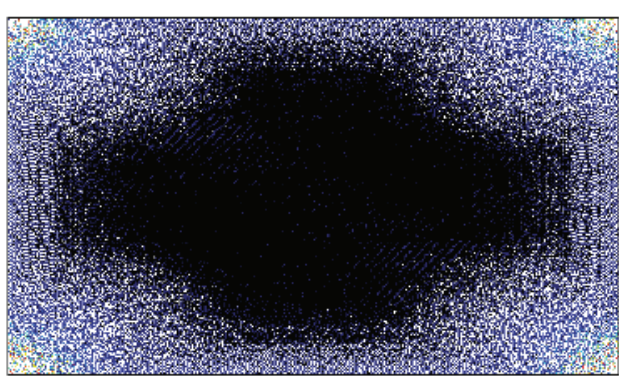

DCT
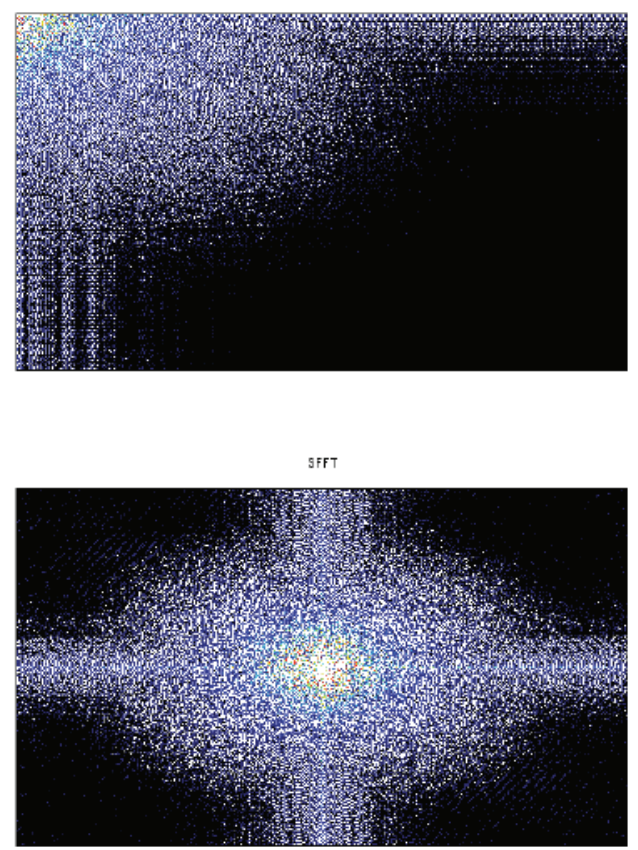

(a)
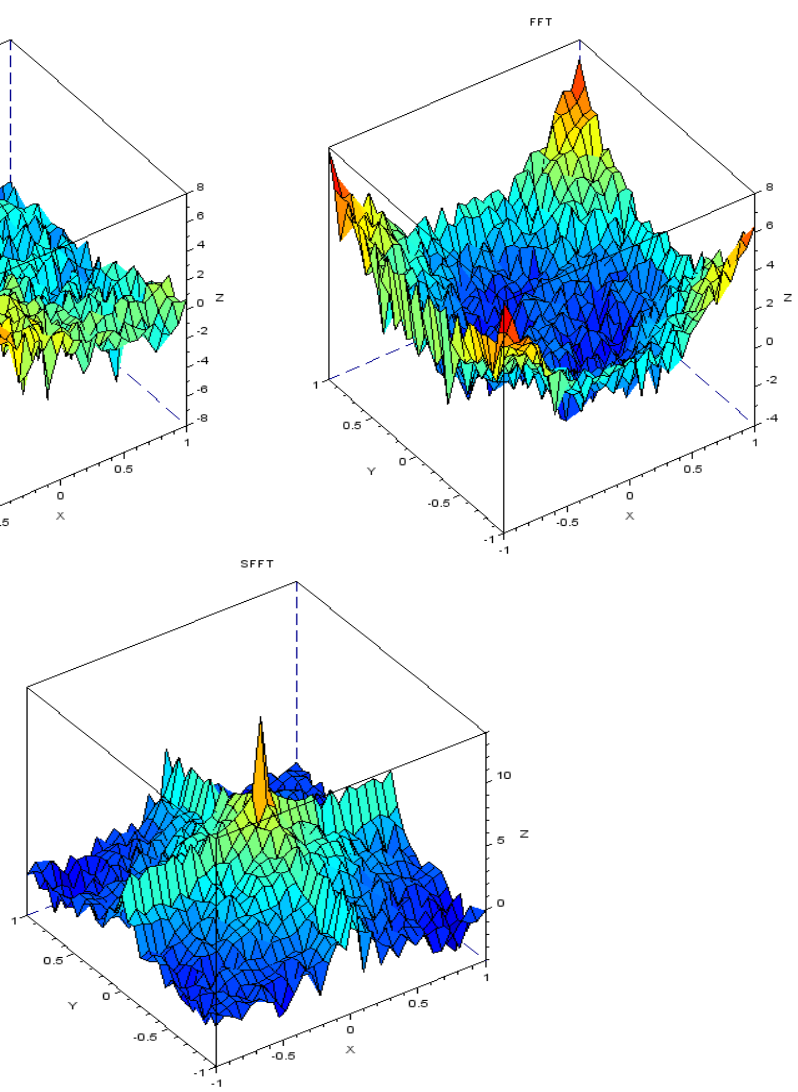

(b)

Figure 6. Results of image with photocopy effect, a) Image with photocopy effect (top-left) and three colormaps of DCT algorithm (top-right), FFT algorithm (bottom-left), SFFT algorithm (bottom-right), b) Frequency domain visualization of DCT algorithm (left), FFT algorithm (right), and SFFT algorithm (bottom) 

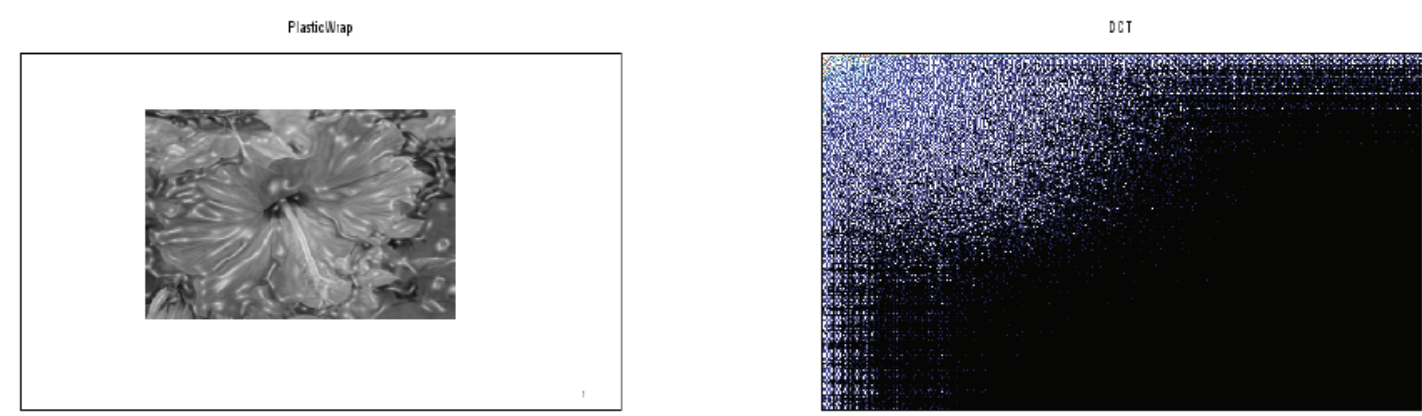

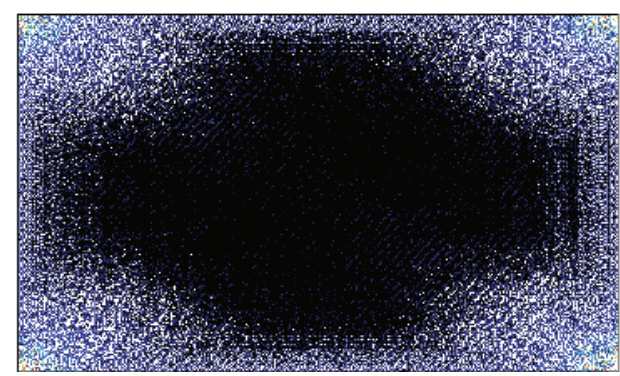

SFFT

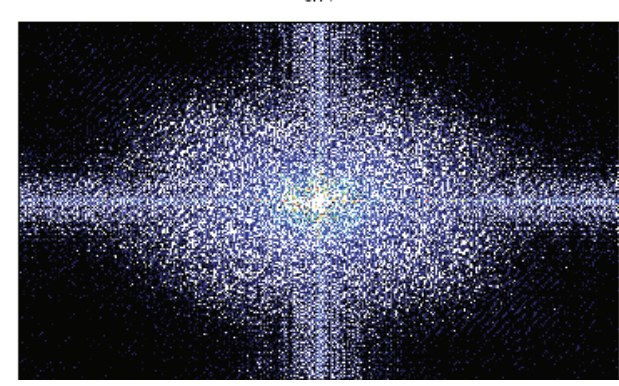

(a)
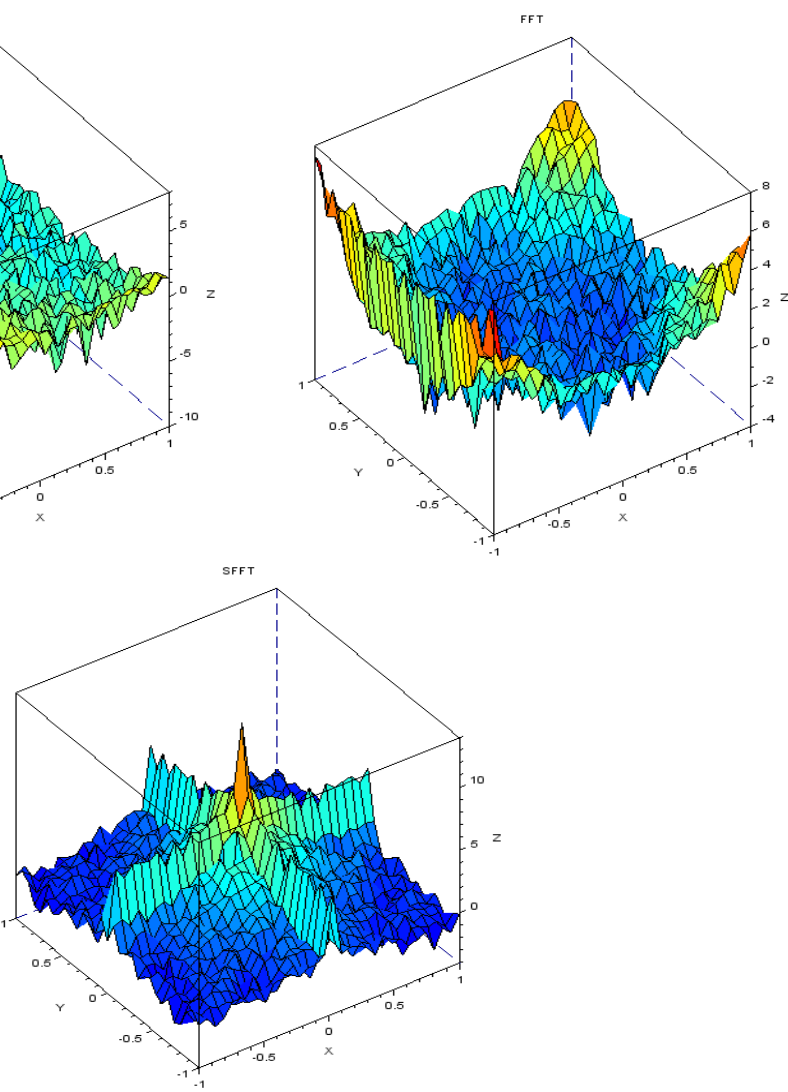

(b)

Figure 7. Results of image with plastic-wrap effect, a) Image with plastic-wrap effect (top-left) and three colormaps of DCT algorithm (top-right), FFT algorithm (bottom-left), SFFT algorithm (bottom-right),

b) Frequency domain visualization of DCT algorithm (left), FFT algorithm (right), and SFFT algorithm (bottom) 


\section{CONCLUSION}

Digital image compression is vital for shape comprehension, storage space and cost-effective transfer. It is useful in practical design of high-speed network environment. This article addresses a comparison of three image compression approaches which are DCT, FFT, and SFFT. The scientific experiments have been undertaken based on images with graphic effect by employing Matlab software. Comparison results regarding colormap as well as frequency spectrum are investigated through visual analysis. Results improve both a shape comprehension of grayscale image and frequency domain convergence in the SFFT algorithm. The graphic output of the SFFT compression algorithm is acceptable, regardless of artistic effect on authentic images. Future research includes the image quality under compression, $\mathrm{CR}$, and security issue on the authentic image.

\section{REFERENCES}

[1] S. Han, et al., "Two-Color Volumetric Imaging of Neuronal Activity of Cortical Columns," Cell Reports, vol. 27, no. 7, pp. 2229-2240, 2019.

[2] A. Kulkarnin and A. Junnarkar, "Gray-Scale Image Compression Techniques: A Review," International Journal of Computer Applications, vol. 131, no. 13, pp. 22-25, 2015.

[3] M. S. Farag, et al., "Parking entrance control using license plate detection and recognition," Indonesian Journal of Electrical Engineering and Computer Science (IJEECS), vol. 15, no. 1, pp. 476-483, 2019.

[4] A. S. A. Salam, et al., "Spatial domain image enhancement techniques for acute myeloid leukemia (M1,M4,M5,M7)," Indonesian Journal of Electrical Engineering and Computer Science (IJEECS), vol. 14, no. 1, pp. 250-257, 2019.

[5] S. Devi and A. Kalia, "Image Compression Using Discrete Cosine Transform (DCT) \& Discrete Wavelet Transform (DWT) Techniques," International Journal for Research in Applied Science \& Engineering Technology, vol. 5, no. 10, pp. 1689-1696, 2017.

[6] R. Koolen, et al., "The Effect of Scene Variation on the Redundant Use of Color in Definite Reference," Cognitive Science Journal, vol. 37, no. 2, pp. 395-411, 2013.

[7] A. J. Coia and M. A. Crognale, "Asymmetric effects of luminance and chrominance in the watercolor illusion," Frontiers in Human Neuroscince, vol. 8, p. 723, 2014.

[8] L. Y. Jie, et al., "Metrics and Benchmarks for Empirical and Comprehension Focused Visualization Research in the Sales Domain," Indonesian Journal of Electrical Engineering and Computer Science (IJEECS), vol. 12, no. 3, pp. 1340-1348, 2018.

[9] Rasiq S. M., Jeevan K.M., S. Krishnakumar., "A Fast-Efficient parallel processing algorithm for straight line detection," Indonesian Journal of Electrical Engineering and Computer Science (IJEECS), vol. 16, no. 3, pp. 1320-1326, 2019.

[10] C. Kanan and G. W. Cottrell, "Color-to-Grayscale: Does the Method Matter in Image Recognition?," PLOS ONE, vol. 7, no. 1, p. e29740, 2012.

[11] A. Güneş, et al., "Optimizing the Color-to-Grayscale Conversion for Image Classification," Signal Image and Video Processing, vol. 10, pp. 853-860, 2016.

[12] C. T. Nguyen and J. P. Havlicek, "Colorto grayscale image conversion using modulation domain quadratic programming," IEEE International Conference on Image Processing, pp. 4580-4584, 2015.

[13] A. H. Ali, et al., "Image and audio fractal compression: comprehensive review, enhancements and research directions," Indonesian Journal of Electrical Engineering and Computer Science (IJEECS), vol. 15, no. 3, pp. 1564-1570, 2019.

[14] A. J. Hussain, et al., "Image compression techniques: A survey in lossless and lossy algorithms," Neurocomputing, vol. 300, pp. 44-69, 2018.

[15] E. R. Arboleda, et al., "Android Application for Microcontroller-based Reservoir Water Level Monitoring," Indonesian Journal of Electrical Engineering and Informatics (IJEEI), vol. 6, no. 1, pp. 79-85, 2018.

[16] L. Lakshmi and E. A. M. Anita, "Run-Length Coding Algorithm Based Satellite Image Compression," Indonesian Journal of Electrical Engineering and Informatics (IJEEI), vol. 5, no. 4, pp. 326-329, 2017.

[17] J. Babbar and N. Rathee, "Satellite Image Analysis: A Review," IEEE International Conference on Electrical, Computer and Communication Technologies, pp. 1-6, 2019.

[18] F. Pabian, "Commercial Satellite Imagery as an Evolving Open-Source Verification Technology," JRC Technical Reports, pp. 1-72, 2015.

[19] A. M. Raid, et al., "Jpeg Image Compression Using Discrete Cosine Transform - A Survey," International Journal of Computer Science \& Engineering Survey, vol. 5, no. 2, pp. 39-47, 2014.

[20] J. Hu, et al., "Image Compression Based on Improved FFT Algorithm," Journal of Networks, vol. 6, no. 7, pp. 1041-1048, 2011.

[21] M. Wang, et al., "Precise and Fast Phase Wraps Reduction in Fringe Projection Profilometry," Journal of Modern Optics, vol. 64, no. 18, pp. 1862-1869, 2017.

[22] A. Bamford and M. Wimmer, "The Role of Arts Education in Enhancing School Attractiveness: A literature review," European Expert Network on Culture, pp. 1-63, 2012.

[23] J. F. Punzalan, "The Impact of Visual Arts in Students' Academic Performance," International Journal of Education and Research, vol. 6, no. 7, pp. 121-130, 2018. 
[24] K. Masaoka and Y. Nishida, "Metric of color-space coverage for wide-gamut displays," Optics Express, vol. 23, no. 6, pp. 7802-7808, 2015.

[25] M. Tehrani, et al., "Artistic Instance-Aware Image Filtering by Convolutional Neural Networks," International Symposium on Telecommunications, pp. 710-714, 2018.

[26] K. B. Schloss, et al., "Mapping Color to Meaning in Colormap Data Visualizations," IEEE Transactions on Visualization and Computer Graphics, vol. 25, no. 1, pp. 810-819, 2019.

[27] C. J. Satish and A. Mahendran, "The effect of 3D visualization on mainframe application maintenance: A controlled experiment," Journal of King Saud University - Computer and Information Sciences, vol. 31, no. 3, pp. 403-414, 2019. 